\title{
Grande Guerra. Al fronte e dietro il fronte
}

\author{
Claudio MAGRIS \\ Università di Trieste $^{1}$ \\ rpacor@yahoo.it \\ Giovanna IOLI \\ Università di Torino \\ giovanna.ioli@tin.it
}

Recibido: 30/04/2015

Aceptado: 30/06/2015

\section{RIASSUNTO}

Due voci per mettere in campo i temi del nuovo Adamo e dell'illusione di pace perpetua, il tema del suicidio d'Europa, la contraddizione fra la bestialità dei conflitti e i valori di solidarietà che pur in essi fioriscono. Due voci per aprire anche finestre nel buio della Prima Guerra Mondiale, dietro il fronte, dove scorrono letteratura e vita di chi fa la Storia e di chi la subisce, dove fioriscono amicizie e malattie da combattere, coraggio e tenerezza di famiglie confinate nel silenzio e nell'ombra.

Parole chiave: Prima Guerra Mondiale, fronte, Europa, crisi.

\section{The Great War. At the front and behind the front}

\begin{abstract}
Two voices discuss the theme of the new Adam and the illusion of the eternal peace, the topic of the suicide of Europe, the contradiction between the brutality of the war and the solidarity, which exists, in spite of everything, into the war itself. Two voices observe the darkness behind the front, where, during the First World War, the literature and the life of the agents and of the victims of the history developed, and where the friendship and the illness the people had to confront, the courage and the affection of families hidden in silence and in the shadow blossomed.
\end{abstract}

Key words: First World War, front, Europe, crisis.

SOMMARIO: 1. Il nuovo Adamo (Claudio Magris) 2. Inutile strage (Claudio Magris) 3. Il fronte della famiglia (Giovanna Ioli) 4. La guerra e le malattie (Giovanna Ioli) 5. Guerra e fraternità (Claudio Magris) 6. Gabriele D'Annunzio: i grissini e il lauro

${ }^{1}$ Poiché i due autori non sono più in servizio attivo presso le istituzioni universitarie di afferenza, rimandano agli indirizzi di posta elettronica come recapito utile. 
(Giovanna Ioli) 7. Sogni di pace germi di guerra (Claudio Magirs) 8. Suicidio d'Europa (Claudio Magris) 9. Nota sugli inediti.

\section{IL NUOVO ADAMO (Claudio Magris)}

A Prosecco, sul Carso vicino a Trieste, c'è un piccolo cimitero austroungarico della prima guerra mondiale. Ne ho parlato e scritto spesso, perché esso è collegato - almeno per me - a una piccola storia che rivela tutta la candida ingenuità con cui l'Europa, con la Grande Guerra, si avviò al grande massacro e - come disse Benedetto XV, uno dei pochissimi ad aver visto con chiarezza la realtà - al proprio suicidio.

In quel cimitero è sepolto un ufficiale austriaco, padre del grande storico viennese Adam Wandruszka. Come mi raccontò lui stesso, egli si chiamava Adam perché sua madre era incinta quando il padre era partito per il fronte, dicendo che, se fosse nato un maschio, avrebbero dovuto dargli il nome di Adam. Da quella guerra, diceva con convinzione poco prima di morire sul Carso, sarebbe nato l'uomo nuovo, fraternamente amico di tutti gli altri, perché dopo quella guerra non ce ne sarebbero state mai più altre e il mondo sarebbe divenuto - o ritornato - un Paradiso Terrestre. Su tutti i fronti avversi, i quali si apprestavano a scannarsi, si pensava e si sentiva, almeno in grande maggioranza, allo stesso modo. La poesia russa di quegli anni è piena dell'attesa di un "uomo nuovo", libero cittadino in un mondo di pace e di vita rinnovellata e in tutte le letterature si trova questa sensazione di una rigenerazione - non soltanto guerresca e bellicosa, come la intendevano i nazionalisti, ma anche nobilmente e fraternamente spirituale - che sarebbe seguita alla guerra. Tranne il lungimirante o almeno lucido pontefice e alcuni socialisti e anarchici pacifisti - mossi peraltro da sentimenti umanitari più che da un preciso giudizio storico - tutti auspicavano o almeno accettavano la guerra. Ogni paese pensava di dare una piccola bella lezione al nemico più vicino, ricavandone vantaggi territoriali o d'altro genere e non riuscendo a immaginare che le ostilità, da quasi tutti viste come un fenomeno di breve durata, potessero assumere dimensioni mondiali e una durata senza fine. I nazionalisti di ogni paese vagheggiavano accrescimenti di potenza grazie alla facile vittoria; molti liberali si attendevano la correzione di antiche ingiustizie; i comunisti vedevano nella guerra la possibilità della rivoluzione; i democratici sognavano l'abbattimento di tutte le frontiere, la fine di regimi autoritari e un dialogo fraterno fra i popoli.

Nessuno riusciva a immaginare - l'immaginazione umana è assai debole, e incapace di pensare una realtà diversa da quella del momento - che la guerra potesse essere così tremenda, specialmente per le truppe al fronte, e averne una tale durata. 


\section{INUTILE STRAGE (Claudio Magris)}

La strage fu, a parte ogni altra considerazione, veramente "inutile", come la definì Benedetto XV, perché alla fine di quella carneficina i problemi che l'avevano scatenata rimasero irrisolti e ancora più acuti. I massacri di Verdun, del Carso, di Leopoli («tomba di popoli») lasciarono un'Europa devastata e avvelenata dai vecchi problemi - i confini, le nazionalità, il nuovo ruolo delle masse, i conflitti sociali ancora più gravi. In quel nuovo Eden in cui avrebbe dovuto vivere felice e buono il nuovo Adamo vennero a regnare e a incrudelire Mussolini, Hitler, Stalin e altri despoti in altri paesi; sulle rovine degli imperi nacquero regimi totalitari, liberticidi e assassini. La fine della prima guerra mondiale creò un'Europa inevitabilmente avviata a una seconda guerra mondiale o meglio a un secondo tempo dell'unica guerra mondiale, che ha visto pure un terzo tempo (per nostra egoistica fortuna giocato in terre lontane da noi) con la guerra fredda, 45 milioni di morti fra il 1945 e il 1989. Può darsi si profili o sia già in atto un quarto tempo, già serpeggiante, anch'esso sottratto alla miopia della intelligenza umana e soprattutto politica. La guerra in Afganistan sta già durando due volte e mezzo la seconda guerra mondiale e non può essere né vinta né persa ossia forse non può finire e questo è solo un esempio.

\section{IL FRONTE DELLA FAMIGLIA (Giovanna Ioli) ${ }^{2}$}

Le parole di autori italiani che trovarono la morte o sopravvissero alla Grande Guerra compongono un elenco entrato ormai a far parte di una coralità antologica ancorata nella memoria: la «bocca / digrignata / volta al plenilunio» di Ungaretti (1969: 25, vv. 5-7); la mancanza di un «bene che paghi la lagrima pianta invano» di Serra (2015: 60); i ricordi olfattivi di sangue, cuoio, anice e d'erba «annusata la faccia contro la terra, spiando la piega del terreno-riparo per il prossimo balzo» di Solmi (1984: 62); il «frammisschiamento di fatterelli e tragedia» e gli errori strategici o la retorica per incitare i soldati a morire senza neppure comprenderne il motivo annotati da Gadda (2008: 292). Sono le montaliane «faville di un tirso» (Montale 1984: 61, v. 22) nel calderone di una Storia che riuscì ad ammutolire chi partì come Clemente Rebora, vagheggiando parole che avessero l'effetto di un colpo di mitraglia e, dopo tanta «melma e sangue» (Rebora 2008: v. 5), tornò intriso di cristianesimo e vocazione al silenzio. Nel ventaglio di prospettive di ogni ricorrenza bellica c'è tuttavia una parte destinata a restare sempre fuori scena, considerata minore perché rientra nell'ambito di una quotidianità esclusa dalla Storia. Sono considerazioni affrontate da Paul Ginsborg nel poderoso volume Famiglia Novecento. Vita familiare, rivoluzione e dittature 1900-1950, dove

\footnotetext{
${ }^{2}$ Riguardo agli inediti del carteggio fra Marianna Montale e Minna Cognetti presentati in questo e nel successivo paragrafo quattro, si veda la Nota che chiude il presente scritto.
} 
osserva che nessuna analisi storiografica ha mai posto al centro le famiglie. ̇̀ sorprendente, scrive, come nella gran maggioranza degli studi sul XX secolo queste restino perennemente dietro le quinte, separate dal solco che divide da sempre il mondo di chi fa la Storia da chi la subisce. Perciò la lettera che Renato Serra scrisse dalla trincea alla madre nel pomeriggio del 20 luglio 1915, poco prima di essere colpito in fronte da un proiettile, non appartiene alla storiografia, ma a «niente di nuovo: le solite vicende di temporale e di sole, e lo spettacolo di un'azione che s'intravede e si sente rumoreggiare sui monti circostanti» (Serra 1920: 200). Paradossale resta la storia del Rifugio di Federico de Roberto, dove un codardo disertore poi fucilato si rivela nei racconti familiari come il più puro degli eroi. Centinaia di lettere che parlano di parenti al fronte sono finite al macero, ma quelle che parlavano di un ragazzo del 1896 destinato al Nobel si salvarono. Fu lui, Eugenio Montale, dopo mezzo secolo a raccontare in un'intervista a Giorgio Zampa che la sorella era «una specie di Eugénie de Guérin» e che i suoi carteggi «esistono ancora» (Montale 1996: 1723). Marianna infatti, scriveva quasi quotidianamente alle amiche, annotando in ogni dettaglio umori, incontri, letture, studi e rapporti, con particolare attenzione ai quattro fratelli maschi. In quella sorta di diario, attento anche agli aspetti sociali del suo tempo, le prime inquietudini per la guerra che già mieteva vittime oltre confine si affacciano il 6 febbraio 1915, quando in casa comincia a serpeggiare una sorta di malumore:

Mammà - scrive - è così scoraggiata e parla sempre di morire, Papà è molto nervoso per tanti contrattempi negli affari, causa le complicazioni internazionali. Ugo, Alberto ed Eugenio sono anch'essi in un momento niente buono. Alla sera dopo aver pranzato non posso ritirami in camera, sapendo di tante nuvole.... Sento che devo stare con gli altri per capire, per non lasciarmi sfuggire nulla. (Marianna Montale)

Due mesi dopo, l'illusione della neutralità dell'Italia è già sfumata e le prime cartoline di reclutamento arrivano anche a casa loro, dove i timori sono soprattutto per Eugenio, «così gracile, pallido, nervoso! E ora li pigliano anche delicati. $\mathrm{Ne}$ hanno preso uno che è tisico dichiarato» (Marianna Montale). Allora Montale trascorreva il suo tempo in biblioteca, contagiato da quella sorella onnivora di libri, ma anche animata dal senso del dovere che faceva sentire giusta anche l'eventualità che i suoi fratelli fossero pronti a combattere. Le piazze di Genova cominciarono a riempirsi di manifestanti e il 14 maggio lei scrive all'amica Minna Cognetti che:

il Re ha confermato Salandra e ha firmato il decreto di mobilitazione. Che Dio illumini i governanti! Che Dio benedica i nostri soldati! Dolori, strazi, orrori....è vero, è tutto vero, ma può esserci un perché anche in questo, se Dio lo permette. Solo il far male è male. Morire bisogna lo stesso. Meglio morire per fedeltà al nostro dovere, in uno slancio di ideale. Credi, vorrei essere un uomo anch'io. (Marianna Montale)

Le sue certezze patriottiche sembravano incrinarsi solo pensando al più giovane di casa e in fondo sperava che potesse essere riformato, dandogli il tempo di 
crescere e irrobustirsi fino a diventare come gli altri. Il 9 giugno confidava all'amica:

Che strana bizzarra, vivace, sarcastica intelligenza ha Eugenio! È senza dubbio il più intelligente di tutti. Salvatore lo chiama "lo spirito che nega"" perché è sempre così ironico e diffidente e scettico. Scettico apparentemente, ma assai mistico e spirituale in fondo; crede quasi più nell'invisibile, nel soprannaturale che in ciò che vede. (Marianna Montale)

Nel frattempo, i fratelli maggiori partivano uno dopo l'altro per il fronte, Alberto in Trentino, Ugo sul Carso, Salvatore a S. Maurizio Canavese, mentre Eugenio restò in attesa di passare fino al settembre 1917, quando fu finalmente dichiarato abile e andò a raggiungere il $23^{\circ}$ fanteria di Oleggio (NO) con Marianna a fianco, pronta a fermarsi qualche giorno per valutare il suo stato d'animo.

Mi par di vedere, a volte, in Genio, la paura di rimbecillire, l'angoscia di perdere del tempo prezioso. Non più leggere e scrivere - non più libertà di cogliere a volo l'ispirazione ... Perché scriveva, sai, delle poesie così strane, incomplete ma geniali, in cui c'era realmente qualche cosa. Se avesse un compagno della sua condizione da poter scambiare qualche parola! Ma nella sua compagnia non ce ne sono. Qui all'albergo vengono alla sera a pranzo due ragazzi di Milano del '99 - ma non mi sembrano tipo da dirsela con Genio. Son quei soliti tipi di ragazzi, un po' vuoti, che fanno da giovanotti. Genio è troppo serio e filosofo! È speciale. E appunto per questo soffre di più di quello che soffrirebbe ogni altro al suo posto. Anche perché è così nervoso! $\mathrm{E}$ io sento come lui e più di lui quello che gli passa nell'anima... Soffro, ma accetto tutto. E ho fiducia che a Genio farà bene la vita militare, gioverà moralmente - perché era troppo sognatore, passivo, sulle nuvole, inadatto alla vita pratica; a furia di pensare perdeva l'energia per qualunque cosa, si rovinava. (Marianna Montale)

Il contrasto tra letteratura e vita era piombato sulle loro vite come un fulmine. A Genova c'erano già tredici ospedali militari e anche le suore dell'Istituto Ravasco avevano convertito il loro magnifico palazzo, scriveva Marianna, pronta a scendere in campo e curare le ferite di quei soldati che ormai si ammassavano in ogni angolo del paese.

\section{LA GUERRA E LE MALATTIE (Giovanna Ioli)}

Marianna Montale aveva una predisposizione naturale per le cure di corpi e anime e quando Eugenio passò a Parma per frequentare un corso accelerato per allievi ufficiali, la sua ansia crebbe, perché conosceva la sua sensibilità e temeva che il disagio psicologico davanti al massacro in corso potesse diventare patologico. Scriveva all'amica che «Genio sta bene di fisico - perché lo zaino non lo porta (è esonerato dal medico) e nelle marce ha sempre resistito (anche a casa). Ma soffre per tante cose!» (Marianna Montale). Il 22 dicembre ripete che suo fratello ha un fisico piuttosto «scalcinato» ma tutto sommato sano, soffre solo «di una 
ipersensibilità nervosa e di "malattie filosofiche" come egli dice - ma sai che è il peggior soffrire» (Marianna Montale). Quello che, tuttavia, la preoccupa maggiormente è il ruolo che assumerà come ufficiale, perché è convinta che quando dovrà condurre i suoi uomini alla baionetta, non potrà mai impartire l'ordine di uccidere. Perciò spera segretamente che il medico si accorga della sua particolare sensibilità, ma il 23 gennaio 1918 lui dichiara che non soffre di alcuna malattia che possa esentarlo dalla guerra, solo «oliquemia ed esaurimento nervoso», insufficienti per l'inabilità, ma Marianna non è convinta, perché:

per tre volte fu fatto rivedibile da medici militari e la quarta volta fu tenuto all'ospedale in osservazione prima di esser preso. Dunque....vuol dire che anche agli occhi dei medici non risulta un fisico da guerriero. Ma quello che io temo per lui è la non resistenza nervosa. (Marianna Montale)

Aveva intuito con largo anticipo la possibilità, poi teorizzata da Freud nel 1920, prima d'allora descritta come un esaurimento del combattente, che non permette alle difese immunitarie di lottare contro germi infettivi vecchi e nuovi: tubercolosi, tifo petecchiale, polmoniti e meningiti, congiuntiviti granulose e così via. Queste notizie circolavano in piccole pubblicazioni con consigli "per l'igiene di guerra", elencate dal capitano medico della Regia Marina Carlo Muzio, che non cita i sintomi psicopatologici della nevrosi vera e propria, forse perché a quei tempi erano catalogati come "codardia", per la quale era prevista una sola cura radicale, la fucilazione. Marianna era anche preoccupata per la predisposizione di Eugenio ai geloni, che potevano diventare lesioni di un'altra patologia da Grande Guerra, quella chiamata "piede da trincea", che esigeva cure immediate perché spesso determinava rimedi altrettanto radicali, come l'amputazione dell'arto.

I primi giorni di aprile Eugenio partì con il $158^{\circ}$ reggimento della Brigata Liguria per aggiungersi ai soldati delle trincee in Vallarsa e, pochi mesi dopo, sarà in prima linea sotto il Monte Corno, poi ancora alle pendici del Pasubio lungo la linea di confine delle fortificazioni austriache, dove si aggirava anche Hemingway al volante dell'American Red Cross. In trincea:

Tacevano gli spari, nel grembo solitario

non dava suono che il Leno roco.

Sbocciava un razzo su lo stelo, fioco

lacrimava nell'aria.

Le notti chiare erano tutte un'alba

e portavano volpi alla mia grotta.

Valmorbia, un nome - e ora nella scialba

memoria, terra dove non annotta. (Montale 1984: 43, vv. 5-12)

Sono questi gli unici versi che scrisse in quei giorni e li aggiungerà agli Ossi di seppia nel 1925. Miracolosamente i quattro fratelli torneranno a casa indenni, fatta eccezione per la granata che incrociò il 2 luglio 1918 il fratello Salvatore durante 
una perlustrazione nelle linee nemiche, ferendolo a una coscia e a un avambraccio. La stessa sorte toccò allo scrittore di Addio alle armi, che seppure ferito cercò di portare in salvo un commilitone più grave di lui. Sarebbe rimasto zoppo per tutta la vita, spiega Giorgio Cosmacini in Guerra e medicina. Dall'antichità a oggi, ma le radiografie, appena inventate, «consentirono di valutare bene la ferita e la riabilitazione, fatta al padiglione Ponti di Milano, di prevenire la zoppia. Addio alle armi è figlio dei progressi della medicina» (Cosmacini 2011: 175). Il 15 marzo 1919 i cinque fratelli Montale si riuniranno tutti a Genova per tributare le «meritate onoranze» a quella sorella speciale, «come se la guerra - commentò lei divertita l'avessi fatta io, invece di loro!» (Marianna Montale).

\section{GUERRA E FRATERNITÀ (Claudio Magris)}

Naturalmente anche la guerra conosce e forse contribuisce a creare valori umani: la lealtà, la fedeltà, il coraggio, la solidarietà verso il compagno in difficoltà anche a prezzo del pericolo e talora l'umana solidarietà pure nei confronti del nemico, almeno quando il nemico ha il volto preciso di un individuo in difficoltà e minacciato dalla morte. La poesia nata dal dolore della guerra è una poesia umanissima, che spesso tocca le corde più universali dell'umano. Si pensi - ma sono soltanto pochi esempi fra molti - a Ungaretti, alla Buffa di Camber Barni che ha espresso il nesso profondo e struggente, epico, malinconico e sanguigno, tra la guerra e la vita; si pensi alle grandissime e fosche poesie di Trakl; a tanti romanzi come quello famoso di Remarque o a tanti film come La grande illusione di Renoir, anch'essa pervasa dal doloroso sentimento della ineluttabilità della guerra e del suo legame indissolubile ancorché doloroso con la vita, sentimento presente soprattutto nei ceti popolari e contadini, in quell'umanità abituata a sentir passare sopra di sé il vento della storia accettata come si accetta la pioggia o l'inverno. Sono questi uomini i veri protagonisti della guerra, vittime ed eroi oscuri, non certo i marescialli che li mandavano a morire senza nemmeno conoscere la cosiddetta arte della guerra, come il nostro Cadorna, al quale è piuttosto inopportuno che sia dedicata una via a Trieste.

C'è una grande letteratura di testimonianza, di diari di guerra, che ha dimostrato come anche nell'inferno possa sopravvivere la nobiltà dell'uomo. Libri come il terribile Un anno sull'altopiano di Lussu, forse il più forte, il più grande nella sua spietata ancorché fraterna denuncia; libri come Guerra del '15 di Stuparich o come quelli di Gadda e di altri. Le testimonianze più preziose sono quelle dei patrioti antinazionalisti, capaci di vedere l'umanità nel nemico, come ad esempio in quello straordinario episodio in cui una compagnia (o altra piccola unità militare, non ricordo esattamente) guidata da Guido Devescovi, l'amico di Slataper e il germanista che per primo scrisse sul Doktor Faust di Thomas Mann, conquistò una postazione austriaca a cento metri di distanza - cento metri la cui conquista, perdita e riconquista era costata la distruzione quasi totale della compagnia italiana, arrivata a quella quota in cinque o sei uomini, e la distruzione totale della compagnia 
austriaca, ridotta a uno solo, un tenente o sottotenente che i soldati, imbestialiti dal macello, stavano probabilmente per uccidere e che Devescovi dovette fermare puntando contro di loro la sua pistola. E così, da quelle rocce e da quelle feritoie insanguinate poté nascere un fiore di amicizia, come quella tra Devescovi e l'ufficiale austriaco.

Fra i caduti per Trieste italiana c'è anche mio zio Galileo, morto a 18 anni volontario sul Carso, mentre un altro zio più anziano, Virgilio, ha fatto la Grande guerra da regolare ufficiale degli Alpini, perché mio nonno Sebastiano, venuto a Trieste dal Friuli, non aveva mai voluto abbandonare la cittadinanza italiana. E così nel '15 la famiglia si era trasferita a Pistoia.

Altro esempio di nobiltà è stato offerto da alcuni, specialmente intellettuali, italiani, profondamente contrari all'entrata in guerra dell'Italia e anche, in certi casi, attivi nel cercare di influire in tal senso sull'opinione pubblica e sulla politica ma che, quando l'Italia entrò in guerra, ai loro occhi sciaguratamente, sentirono - come il grande filologo Cesare De Lollis, ma è solo un esempio fra i tanti - il dovere di arruolarsi volontariamente, di condividere, nel bene e nel male, il destino della loro patria.

Dalla guerra nacque certo il fascismo, ma anche il fiore di un antifascismo resistente. Un solo esempio fra i molti: Ercole Miani, eroico volontario e poi con D'Annunzio a Fiume, il quale nella Trieste occupata dai nazisti fu a capo della resistenza democratica e orribilmente torturato dalle belve della banda Collotti, provvidenzialmente fucilate poco dopo, non si lasciò scappare una parola e non menò mai vanto del suo autentico eroismo.

La guerra ha prodotto anche fiumi di esaltazione feroce e sconsiderata, una letteratura esaltata dalla violenza. Forse il vertice della bestiale e imbecille disumanità è stato raggiunto da una pagina di Giovanni Papini il quale (senza peraltro aver combattuto, a differenza di molti suoi amici) si compiaceva del sangue delle plebi ossia dei soldati che, così scriveva, concimavano e rendevano fertile la terra e, toccando l'apice della volgarità e dell'inumanità, aggiungeva che non gli importava che gli venissero a dire che le madri di tutti quei caduti sconosciuti avevano pianto e piangevano, perché «non piansero quando si incinsero». Rimproverare a una madre che piange la morte del figlio il fatto di non aver pianto mentre lo concepiva è una bestialità che ha pochi confronti.

Anche l'amore di patria è un profondo valore, specie se lo si intende come Mazzini inserito nell'amore più vasto per un coro più grande di patrie, legate da valori comuni e sentimenti fraterni. È giusto commuoversi sentendo cantare «cimitero di noi soldati / forse un giorno ti vengo a trovare» o anche «Lili Marlene», cantata soprattutto, contrariamente a quello che si crede, nella prima guerra mondiale. C'è qualcosa di grande nei sogni dei diversi irredentismi, illusi di poter convivere fraternamente. C'è anche qualcosa di grande nella disponibilità di molti patrioti a morire, come ricorda in una splendida pagina Elody Oblath Stuparich, l'amica di Slataper e poi moglie di Giani Stuparich; lei stessa però, in questo splendido ricordo che dice tutta l'umanità di quella generazione, dice che 
erano pronti non solo a morire, ma anche alla morte di milioni di uomini, pur senza rendersene conto.

\section{GABRIELE D'ANNUNZIO: I GRISSINI E IL LAURO (Giovanna Ioli) ${ }^{3}$}

Il primo giugno 1929 Gabriele D'Annunzio, ormai confinato come ospite o prigioniero del Duce al Vittoriale, spedisce un telegramma a Nino Amilcare Vaudano e Pierina Rivero che si chiude «nella gloria dei santi Pipino e Fino. Stop». Voleva semplicemente ringraziare per quelli che, in un'altra lettera, chiama gli «assidui grissini», rinnovando sull'altare di quel fornaio torinese la fedeltà a un ideale condiviso nella carta del Carnaro dieci anni prima. Il 15 aprile 1926 era stato più esplicito e ornato nell'attribuire al suo corrispondente la «gentilezza fiumana» che, «come un fiore azzurro intrecciato alla quercia e al lauro», li aveva nutriti e ora si esprimeva con doni mangerecci per un «Comandante sano e ancora di più quando è infermo». Dal suo eremo D'Annunzio vagheggiava altri voli non solo poetici, manifestando il desiderio di raggiungere Torino anche «per una visita aviatoria. Ho nomato 'Campo della Promessa', intanto, il campo di Lonate. Quale è, o uomo adriaco, la Promessa? Ti mando un ritratto di guerra, che ho scoperto in questi giorni tra molte carte grevi» (D'Annunzio, inedito). Da una promessa di servire la Patria e liberare Fiume era nata anche la loro amicizia. Nino, classe 1879, figlio di Antonio che si era guadagnato cinque medaglie nella spedizione dei Mille, cominciò a collezionare le sue a soli sei anni, vincendo una gara di scherma organizzata dalla gloriosa Ginnastica Torino. Pochi anni dopo il re Umberto, ospite d'onore di una manifestazione sportiva, incuriosito da quel ragazzino minuto che aveva tre file di medaglie sul petto, gli chiese per quale disciplina le avesse meritate e lui elencò una serie di specialità agonistiche che il sovrano interruppe con una battuta in piemontese: «Ma ti 't tachi propri a tutt!», ma tu ti attacchi proprio a tutto! Il cimento invernale era certamente la più singolare delle sue prodezze sportive. L'idea era venuta nel 1895 allo scultore Giuseppe Cantù, anima del nuoto milanese e fondatore della Rari Nantes, che invitava i più coraggiosi a tuffarsi nelle acque del Naviglio Grande nei giorni della merla. Nino per quattro o cinque anni partì in bicicletta da Torino solo per mettersi alla prova, poi decise di fondare una Rari Nantes sulle rive del Po, dove ancora oggi si celebra lo stesso rito. Non c'era alcuna inclinazione superomistica in queste gesta sportive, ma la volontà di essere sempre pronti a intervenire in casi estremi, che lui affrontò salvando un soldato impigliato sotto un cavallo di frisia nel lago di Garda, domando un destriero imbizzarrito in una strada affollata, ma anche insegnando a nuotare gratuitamente a pompieri, carabinieri e guardie della sua città. Con questo stesso «spirito» non esitò ad arruolarsi nel Battaglione Ufficiali delle disciolte Milizie Legionarie Fiumane

${ }^{3}$ Riguardo agli inediti dannunziani presentati in questo paragrafo si veda la Nota che chiude il presente scritto. 
dal settembre 1919 al gennaio 1921, dopo avere meritato sul campo della Grande Guerra un'altra fila di decorazioni. Sul petto del capitano degli alpini Nino Amilcare Vaudano brillò così anche la medaglia di bronzo che D'Annunzio instituì per il giuramento «Fiume o morte», coniata per decreto nazionale in memoria del giorno in cui:

dal cimitero carsico dei Ronchi non partì un pugno d'uomini devoti alla morte, un'altra massa di carne da macello, un'altra messe umana offerta alla falce aerea della mitragliatrice. Partì lo Spirito. Per ciò fu irresistibile. Per ciò vinse. Senza colpo ferire. La vittoria del 12 settembre è una vittoria divina. Sine strage vincit. (D'Annunzio, inedito)

Sei anni dopo, il 12 dicembre 1925, gli appuntò sul petto anche una medaglia d'oro, motivandola alla memoria del suo coraggio di quei giorni ormai lontani:

Meraviglioso nelle giornate del Natale di Sangue, più volte traversò le linee di fuoco, ottenendo per noi risultati preziosi. Ovunque, sempre si esplicò con costante prodezza. Fu ed è il Legionario impareggiabile completo esemplare. Carnaro 1919. (D’Annunzio: inedito)

Il 24 aprile 1926 spedisce un lettera anche alla "compagna di Nino Vaudano", vergando sulla busta uno dei suoi motti, «Undique fidus, undique firmus» 'ovunque fedele, ovunque fermo', poi impresso sulla colonna dei giuramenti, al centro dell'Arengo al Vittoriale:

Cara sirocchia, ho riveduto Nino con profonda gioia, rammaricandomi che - nell'assedio implacabile - la mia ora di amicizia sia stata breve. Ma son certo che egli non la dimenticherà. So quanto la vostra anima è bella e forte. Permettete che Mastro Paragon Cappella - l'orafo del Vittoriale - vi offra questi pendenti lavorati con la sua arte solitaria. (D'Annunzio, inedito)

La moglie di Nino Vaudano fu l'altra faccia delle sue medaglie, perché non esitava a raggiungerlo in prima linea, a soccorrerlo quando tornava ferito, a rassicurarlo con dolce fermezza. Perciò dopo, a bocce ferme, quando gli chiedevano dove trovasse tanto vigore, rispondeva che:

il coraggio non è poi una gran cosa. Ho avuto bisogno di un'altra forza, ben maggiore: quella di mantenermi galantuomo in occasioni dove approfittare era facile come ridere e se non approfittavi ti dicevano che eri fesso. Ecco, questa forza me l'ha data mia moglie. (D'Annunzio, inedito)

Quando il sogno di quegli Arditi s'infranse, la vita del Comandante assomigliò più a quella di un prigioniero che di un ospite privilegiato del Duce, diventando uno dei più prolifici autori di lettere e telegrammi: 
Io mi son mostrato, come sempre, esempio di disciplina, rimanendo in silenzio. Noi legionari non possiamo oggi rallegrarci. Io, nell'ora della festa, non potrò non piangere pensando che il Porto di Nazario - dove ebbi la mia Guardia e dove tanto fieramente vissi, di giorno e di notte - il mio Porto è in mano dei porcari odiosi, come la mia Buccari, come la mia Arbe e le altre isole e il Bittorai e il Dinara, ahimè! Sii dunque silenzioso testimone. Va, se puoi, presso il confino di ferro spinato; e conficca in una spina una foglia del lauro del 12 settembre 1919, pensando alla mia tristezza solinga. Nel ritorno passa di qui, sali al Vittoriale, e racconta. La vera gloria è intima, è chiusa, è ben coperta e non dà faville: forse è la mia. (D’Annunzio, inedito)

Nino salì spesso al Vittoriale e con Pierina onorò anche il suo invito per la rappresentazione della Figlia di Iorio e il 10 settembre 1927 lui scrive:

Mio carissimo, allevierai un poco il mio crudele supplizio. Da anni e anni non soffrivo come soffro in questi giorni, neppure nelle peggiori vicende di Fiume. Vieni sulla collina. Al Vittoriale l'entrata per te è libera sempre. Ecco il Gallo, pei pochi che non rinnegheranno. (D’Annunzio, inedito)

Non allude al gallo del tradimento, ma a quello "ognora desto", impresso nei suoi ex libris. La tragedia fu rappresentata all'aperto il giorno dopo, nei giardini del Vittoriale, come spettacolo d'esordio del nuovo Istituto nazionale per il teatro di D’Annunzio, con Maria Melato nel ruolo di Mila e i migliori professionisti per scenografia e costumi. Tra gli spettatori eccellenti di quella sera c'erano anche alcuni protagonisti della storia del teatro novecentesco: Max Reinhardt, Konstantin Stanislavskij, Aleksandr Jakovlevič Tairov e Vsevolod Emil'evic Mejerchol'd.

Dieci anni dopo, un telegramma metterà la parola fine alla loro corrispondenza, testimoniando ancora una volta, come per un malinconico congedo, il miracolo di fedeltà di quel lauro trafitto: «tu non sei il perpetuamente fedele fra tutti, ma sei il simbolo stesso dell'assidua fedeltà - io soffro come non mai - Ti abbraccio e tutti i miei migliori abbraccio in te» (D'Annunzio, inedito). D'Annunzio morirà due mesi dopo, il 1 marzo 1938.

\section{SOGNI DI PACE, GERMI DI GUERRA (Claudio Magris)}

Forse ogni sogno di costruire un nuovo Paradiso, un Eden in cui un nuovo Adamo possa passare la vita a passeggiare liberamente e fraternamente, è pericoloso e finisce per richiedere ecatombi. Forse è meglio accontentarsi di migliorare un po' il mondo, nella misura modesta e concreta delle proprie possibilità, senza lasciarsi intimidire dalle enormi difficoltà che si creano subito dinanzi a qualsiasi progetto anche minimo di rendere migliori le cose, di rendere più umano il mondo. Forse c'è più grande differenza tra i patrioti all'interno di uno stesso campo che fra quelli di un campo e quelli di un altro. C'è più differenza fra $i$ patrioti italiani, anche soprattutto ad esempio triestini, che sognavano un'armonia fraterna tra la cultura italiana e quella slava, come Tommaseo (uno dei padri del 
nostro Risorgimento, che si firmava un "italo-slavo"), e quelli che sognavano il dominio e la repressione del vicino, che non fra un patriota italiano democratico e uno slavo democratico, e ciò vale ovviamente per ogni campo.

Ciò che continua a stupire sempre di più è la mancanza di immaginazione. Riesce difficile capire oggi come si potesse pensare che quella guerra potesse essere simile a quelle del passato, già di per sé sciagurate, senza neanche ipotizzare le proporzioni del macello che si stava preparando. Capisco di più, anche se in aperto dissenso, coloro che, come ad esempio Ernst Jünger, hanno visto nella guerra un elemento formativo dell'uomo e hanno continuato a vederlo anche quando la realtà si è fatta di un'immane brutalità, che non quelli che sono andati in guerra allegramente, pensando che in qualche modo potesse essere pure una "bella guerra"

e poi, dinanzi al massacro all'ingrosso di Verdun, si sono scandalizzati, inorriditi. È proprio necessario fare esperienza di certi mali per poter capire che sono un male? La mia età mi ha risparmiato dall'essere coinvolto in conflitti armati e non ho mai provato concretamente cosa voglia dire ficcare una baionetta nella pancia di un altro o ricevere nella propria pancia la sua baionetta. Ma credo di sapere che si tratti di una cosa orribile e sarebbe strano se immaginassi uno scontro alla baionetta come qualcosa di esaltante, salvo poi scandalizzarmi o peggio quando me ne trovassi coinvolto. Viene in mente quella storia raccontata da Giorgio Voghera: la storia di un ebreo galiziano, cittadino dell'impero austroungarico, richiamato alle armi, che si sottopone a tutto l'addestramento pur restando profondamente avverso a ogni guerra, impara - sempre controvoglia e con i sentimenti più pacifisti del mondo - a sparare o a gettare le bombe a mano, finché, giudicato addestrato in modo sufficiente, viene mandato al fronte, sempre controvoglia e con sentimenti antimilitaristi. Una notte - siamo sul fronte orientale - arriva l'ordine di attaccare le trincee russe situate di fronte. Di malavoglia e sempre contrario, lui obbedisce, esce dalla sua trincea, striscia come gli viene detto sul prato con il suo fucile in mano e puntato nella direzione giusta, finché a un certo punto i russi mandano alcuni razzi che illuminano la zona e cominciamo a sparare sugli austriaci che sono in procinto di attaccare. A questo punto, l'ebreo galiziano si alza e grida, sdegnato: «Ehi, siete pazzi? C'è gente qui!» e cade colpito alla fronte.

\section{SUICIDIO D'EUROPA (Claudio Magris)}

Inutile strage, ma anche suicidio d'Europa. Sino alla grande guerra, l'Europa era stata il centro del potere del mondo, nel bene e nel male; con quel conflitto mondiale, il suo impero è finito e altre potenze hanno cominciato a contendersi il dominio del mondo in una lotta non certo ancora conclusa. Lo sfascio dell'Europa ha permesso a tanti popoli extraeuropei, sino allora sfruttati e oppressi, di liberarsi e di iniziare un cammino, certo tuttora contraddittorio, verso la propria libertà e autonomia.

La prima guerra mondiale quale male necessario per il progresso complessivo dell'umanità? È difficile dire, specie dinanzi all'altissimo prezzo di sangue pagato. 
I nemici, per ognuno dei paesi e dei popoli in guerra, erano pure all'interno di ogni schieramento. Quando fu assassinato a Sarajevo Francesco Ferdinando, erede al trono dell'impero austroungarico, in molti reggimenti ungheresi si brindò gioiosamente alla sua morte. Quando nel maggio 1915 l'Italia entrò in guerra, non molti si accorsero che quella vittoria della piazza sul Parlamento era l'inizio della fine della democrazia, alla cui agonia stiamo forse assistendo.

Ogni guerra è una mina fra le mani di chi si dispone a gettarla. Lo sapeva bene Giovanni Paolo II, che non era un ingenuo pacifista, ma piuttosto, sotto molti aspetti, un rude conservatore, quando si è genialmente ancorché vanamente opposto alla guerra contro l'Islam. Piuttosto vi sono momenti e situazioni in cui può essere inevitabile, necessario e doveroso fare la guerra - come ad esempio dinanzi all'espansione omicida della Germania nazista (la quale peraltro non sarebbe esistita senza la prima guerra mondiale e le inique condizioni del trattato di Versailles). Quando le democrazie occidentali cedettero ignominiosamente a Hitler nel 1938, Churchill disse: «Le democrazie potevano scegliere tra il disonore e la guerra. Hanno scelto il disonore. Non per questo eviteranno la guerra».

Nel 1914 è stato un disonore scatenarla, la guerra, ed è stata un'imbecillità illudersi di poterla controllare e di potersene servire.

\section{NOTA SUGLI INEDITI}

Le lettere citate ai paragrafi 3 e 4, parzialmente inedite, sono tratte dall'epistolario di Marianna a Minna Cognetti, affidato a Giovanna Ioli da Bianca, figlia di Alberto Montale (Cronologia dei fratelli Montale: Salvatore,1885; Ugo, 1887; Ernesto, 1889, morto appena nato; Alberto,1890; Marianna, 1894; Eugenio 1896).

Le lettere inedite di D'Annunzio, citate al paragrafo 6, salvo quelle indirizzate alla moglie, sono state donate dagli eredi di Nino Amilcare Vaudano al Fondo Archivio generale fiumano della Fondazione Il Vittoriale degli italiani. Copie della fotografia citata dal Vate nel paragrafo 6 sono custodite dagli eredi.

\section{RIFERIMENTI BIBLIOGRAFICI}

Cosmacini, Giorgio (2011): Guerra e medicina. Dall'antichità a oggi, Roma-Bari, Laterza.

GADDA, Carlo Emilio (2008): Giornale di guerra e di prigionia. Con il «Diario di Caporetto», a cura di Dante Isella, Milano, Garzanti.

GINSBORG, Paul (2013): Famiglia Novecento. Vita famigliare, rivoluzioni e dittature (1900-1950), Torino, Einaudi.

LUSSU, Emilio (1938): Un anno sull'altipiano, Parigi, Edizioni di Coltura.

MonTALE, Eugenio (1984): Tutte le poesie, a cura di Giorgio Zampa, I meridiani, Milano, Mondadori. 
Montale, Eugenio (1984): «Ho scritto un solo libro», in Eugenio Montale, Il secondo mestiere. Vol. 2: Arte, musica e società, a cura di Giorgio Zampa, I meridiani, Milano, Mondadori, p. 1723.

REBORA, Clemente (2008): Tra melma e sangue. Lettere e poesie di guerra, Novara, Interlinea.

SERRA, Renato (1920): Le lettere. Con l'aggiunta dei frammenti inediti del secondo volume e di un indice onomastico, Roma, Società Editrice La Voce.

SERRA, Renato (2015): Esame di coscienza di un letterato; Carte Rolland; Diario di trincea, edizione critica a cura di Marino Biondi e Roberto Greggi, Roma, Edizioni di Storia e Letteratura.

SolmI, Sergio (1984): «Ricordi del 1918», in Opere di Sergio Solmi 1: Meditazioni e ricordi, a cura di Giovanni Pacchiano, Milano, Adelphi, p. 64.

STUPARICH, Gianni (1931): Guerra del '15. Dal taccuino di un volontario, Milano, Fratelli Treves.

UngaRETTI, Giuseppe (1969): Vita d'un uomo. Tutte le poesie, a cura di Leone Piccioni, I meridiani, Milano, Mondadori. 